\title{
Impulsive Flocking of Dynamical Multiagent Systems with External Disturbances
}

\author{
Fujun Han, Yize Yang, and Hong-Yong Yang \\ School of Information and Electrical Engineering, Ludong University, Yantai 264025, China \\ Correspondence should be addressed to Hong-Yong Yang; hyyang@yeah.net
}

Received 21 April 2016; Accepted 26 December 2016; Published 19 January 2017

Academic Editor: Mohammad D. Aliyu

Copyright (C) 2017 Fujun Han et al. This is an open access article distributed under the Creative Commons Attribution License, which permits unrestricted use, distribution, and reproduction in any medium, provided the original work is properly cited.

Flocking motion of multiagent systems is influenced by various external disturbances in complex environment. By applying disturbance observer, flocking of multiagent systems with exogenous disturbances is studied. Based on the robust features of impulsive control, a distributed impulsive control protocol is presented with disturbance observer, and flocking motion of multiagent systems is analyzed. Moreover, a sufficient condition is obtained to ensure the flocking motion of multiagent systems following a leader. Finally, simulation results show the validity of the theoretical conclusion.

\section{Introduction}

With the development of network communication technologies, coordinated control problems of distributed networked systems have been receiving increasing attention from many research fields, like biology, computer, automation, artificial intelligence, and so forth. As one of the key issues of coordinated control problems, flocking motion has been studied intensively to reveal the natural phenomenon in the form of schooling of fish, flocking of birds, swarming of bacteria, and so on [1-4].

Flocking is a class of the cluster behaviors in which large number of agents are organized into coordinated motion by applying only limited local information and simple rules. Recently, the studies of flocking motion have been researched deeply, and numerous results have been obtained. The classical flocking model with three heuristic rules was proposed by Reynolds [2] in 1980s: (i) Separation: avoid conflicts with nearby flock individuals; (ii) Alignment: attempt to match velocity with nearby flock individuals; (iii) Cohesion: attempt to be close to nearby flock individuals. Based on Reynolds' model, flocking algorithms were proposed by means of a velocity consensus component with a local artificial potential function $[5,6]$. In particular, Olfati-Saber provided a theoretical computational framework to design the flocking algorithms [5]. This framework includes three flocking algorithms: the first one realizes the rules of Separation and
Cohesion in Reynolds' model, the second one is a path tracing algorithm for a group of agents to follow a virtual leader, and the third one embodies the capabilities with obstacle avoidance. The flocking algorithms were studied with an artificial potential function in fixed and switching topologies by Tanner et al. in [6]. By applying a pinning feedback control, flocking control of a group of mobile autonomous agents with a virtual leader was researched by Su et al. [7, 8]. A flocking control problem with the network connectivity was presented with a potential function method in [9]. Based on a bounded potential function, a rendezvous algorithm of multiple agents was studied with preserved network connectivity $[10,11]$. However, these studies worked on the assumption that the disturbances are ignored in multiagent systems.

In the nature applications, interference information exits in all kinetic systems, few individuals have not been affected by the external disturbances. However, when there are uncertainty model parameters and external interference in the motion systems, the dynamical behaviors become more complex and more challenging. Chen [12] pioneered the development of disturbance observers for unknown disturbance, Guo and Chen [13] extended the disturbance observers method from single input single output (SISO) systems to multi-input multioutput (MIMO) models with external disturbances for the first time. Moreover, the disturbanceobserver-based-control method can combine with different control methods according to different requirements [14-16]. 
Based on the idea of disturbance compensation, Yang et al. proposed a consensus algorithm of multiple mobile agents with disturbance-observer-based-control [17, 18].

In this paper, flocking motion of multiagent systems with external disturbances will be studied by applying the impulsive control method. With the effect of incomplete information, external disturbance, and packet loss in network, the dynamic behaviors of the systems will show impulse phenomenon. As an instantaneous behavior, the impulse phenomenon has been widely used in communication systems with switching transmission signal. Zhou et al. studied the synchronization of coupled harmonic oscillators with local instantaneous interaction [19]. Guan et al. proposed impulsive consensus of networked multiagent systems [20]. However, up to now, few researches for the dynamical flocking of multiagent systems with impulsive control have been reported.

The main objective of this paper is to research the flocking motion of multiagent systems by applying the impulsive control method. The innovation of this paper is to apply impulsive control solving the flocking motion of multiagent systems with exogenous disturbances. The rest of the paper is organized as follows. In the second part, problem description and preliminaries are given. The main results are discussed in the third part. In the fourth part, example and simulation results are shown to illustrate the validity of theoretical results. Conclusions are finally drawn in fifth part.

\section{Problem Description and Preliminaries}

2.1. Algebraic Graph Theory. Let $G=(V, E, A)$ be an undirected graph consisting of $V=\left\{v_{1}, v_{2}, \ldots, v_{n}\right\}$ and $E \subseteq$ $V \times V . A=\left[a_{i j}\right]$ is an adjacency matrix of the graph $G$. The relationships among agents are described by the graph without self-loops; that is, $\forall i \in V, a_{i i}=0$. Moreover, the adjacency element $a_{i j}>0$ when $\left(v_{i}, v_{j}\right) \in E$; otherwise, $a_{i j}=$ 0 . The set of neighbors of $v_{i}$ is denoted by $N_{i}=\left\{v_{j} \in V, a_{i j}>\right.$ 0 \}. The Laplacian matrix of the graph $G$ is defined as $L=$ $D-A$, where $D=\operatorname{diag}\left(\operatorname{deg}_{\text {in }}\left(v_{1}\right), \operatorname{deg}_{\text {in }}\left(v_{2}\right), \ldots, \operatorname{deg}_{\text {in }}\left(v_{n}\right)\right)$. Suppose that the eigenvalues of the Laplacian matrix are satisfied with $\lambda_{1}(L) \leq \lambda_{2}(L) \leq \cdots \leq \lambda_{n}(L)$. Then, there are $\lambda_{1}(L)=0$ and $L 1_{n}=\lambda_{1}(L) 1_{n}$, where $1_{n}=[1,1, \ldots, 1]^{T}$. Moreover, if the graph is connected, it has $\lambda_{2}(L)>0$.

2.2. Problem Description. It is assumed that $n$ agents move in two-dimensional Euclidean space with the undirected graph $G$. The dynamic equation of the $i$ th agent is described as

$$
\begin{aligned}
& \dot{q}_{i}(t)=p_{i}(t), \\
& \dot{p}_{i}(t)=u_{i}(t)+d_{i}(t),
\end{aligned}
$$

where $q_{i}(t)=\left(x_{i}(t), y_{i}(t)\right)^{T}, p_{i}(t)=\left(\dot{x}_{i}(t), \dot{y}_{i}(t)\right)^{T}, u_{i}=$ $\left(u_{x i}(t), u_{y i}(t)\right)^{T}$, and $d_{i}(t)$ are the position, velocity, control input, and external disturbance of the $i$ th agent in multiagent systems, respectively. In addition, the external disturbance $d_{i}(t)$ can be illustrated as

$$
\begin{aligned}
& \dot{w}_{i}(t)=A w_{i}(t), \\
& d_{i}(t)=C w_{i}(t),
\end{aligned}
$$

where $w_{i}(t)$ is internal state of the disturbance system; moreover, subsystem matrix $(A, C)$ is observable. Supposing that the state matrix of the controlled system is available, disturbance observe subsystem is expressed by

$$
\begin{aligned}
\widehat{w}_{i}(t) & =z_{i}(t)-Y p_{i}(t), \\
\widehat{d}_{i}(t) & =C \widehat{w}_{i}(t) \\
\dot{z}_{i}(t) & =(A+Y C)\left(z_{i}(t)-Y p_{i}(t)\right)+Y u_{i}(t),
\end{aligned}
$$

where $z_{i}(t)$ is an introduced auxiliary variable and $\widehat{w}_{i}(t), \widehat{d}_{i}(t)$ are the estimation of $w_{i}(t)$ and $d_{i}(t) . Y$, as an unknown matrix, needs to be calculated.

Definition 1. A group of agents asymptotically achieve flocking motion if their velocity vectors will asymptotically achieve consistency and the distances will remain constant between any two agents without collision.

Definition 2. Potential function $U_{i j}$ is a differentiable function of the relative distance $\left\|q_{j}(t)-q_{i}(t)\right\|$ between $i$ node and $j$ node, where $U_{i j}$ is nonnegative, unbounded function and satisfies the following two properties:

(1) Potential function $U_{i j}$ tends to infinity while the distance $\left\|q_{j}(t)-q_{i}(t)\right\|$ tends to zero.

(2) Potential function $U_{i j}$ can get the unique minimum at a desirable distance.

The potential function is applied in paper [5]; for example,

$$
\begin{aligned}
U_{i j}\left(\left\|q_{i}(t)-q_{j}(t)\right\|\right)= & \frac{d^{2}}{\left\|q_{j}(t)-q_{i}(t)\right\|^{2}} \\
& +\ln \left(\left\|q_{j}(t)-q_{i}(t)\right\|^{2}\right),
\end{aligned}
$$

where $d>0$ is a real number. Let $F(z)=d^{2} / z^{2}+\ln \left(z^{2}\right)$; thus $f(z)=d F(z) / d z=2\left(z^{2}-d^{2}\right) / z^{3}$. It is known that potential function $U_{i j}$ can obtain a unique minimum value when $\left\|q_{j}(t)-q_{i}(t)\right\|=d$.

Suppose the dynamics of the leader is described as

$$
\begin{aligned}
& \dot{q}_{r}(t)=p_{r}(t), \\
& \dot{p}_{r}(t)=0,
\end{aligned}
$$

where $q_{r}(t)=\left(x_{r}(t), y_{r}(t)\right)^{T}$ and $p_{r}(t)=\left(\dot{x}_{r}(t), \dot{y}_{r}(t)\right)^{T}$ are the leader's position and velocity. In this paper, the leader will keep a uniform movement. In order to solve the flocking 
motion, control protocol $u_{i}$ is proposed as

$$
\begin{aligned}
u_{i}= & \sum_{j \in N_{i}} a_{i j} f\left(\left\|q_{j}(t)-q_{i}(t)\right\|\right) \vec{n}_{i j} \\
& +k \sum_{j \in N_{i}} a_{i j}\left(p_{j}(t)-p_{i}(t)\right)-\widehat{d}_{i}(t) \\
& -h_{i} c_{2}\left(p_{i}(t)-p_{r}(t)\right) \delta\left(t-t_{k}\right),
\end{aligned}
$$

where $f(z)$ is the same as mentioned above and $\vec{n}_{i j}=\left(q_{j}(t)-\right.$ $\left.q_{i}(t)\right) /\left\|q_{j}(t)-q_{i}(t)\right\|$ is a unit vector. $k \geq 0$ and $c_{2} \geq 0$ parameters will be selected. If $h_{i}=1$, then the $i$ th agent can get information from the leader, while the agent can not get the information from the leader if $h_{i}=0 .\left\{t_{k}\right\}_{k=1}^{+\infty}$ will satisfy $t_{k}-t_{k-1}=T>0$ and $\lim _{k \rightarrow \infty} t_{k}=+\infty . \delta(t)$ is the Dirac function, which activates the impulsive control between agent and leader at the moment of $t_{k}$. If a group of agents always meet $\lim _{t \rightarrow \infty} p_{j}(t)=p_{i}(t)=p_{r}(t)$ and $\lim _{t \rightarrow \infty} q_{j}(t)-q_{i}(t)=a>0$ with any initial state, it is said that multiagent systems achieve the flocking motion with distributed impulsive control.

Remark 3. In this paper, flocking motion problem of multiagent systems with exogenous disturbances will be studied, it is more complex than the problem in reference [5] where the systems are running without disturbances.

\section{Main Results}

Theorem 4. Suppose that n identical agents constitute the fixed topology. Consider the multiagent systems (1) with the external disturbances (2). When the control protocol is continuous with $\delta\left(t-t_{k}\right) \equiv 1$ in (6), if there are appropriate dimensional matrix $P>0$ and $Q$, satisfying the following equation:

$$
\begin{aligned}
& {\left[\begin{array}{cc}
-I_{2} \otimes\left(k L+c_{2} H\right) & \frac{1}{2}\left(I_{2 n} \otimes C\right) \\
\frac{1}{2}\left(I_{2 n} \otimes C^{T}\right) & I_{2 n} \otimes\left(P A+Q C+(P A)^{T}+(Q C)^{T}\right)
\end{array}\right]} \\
& \quad<0,
\end{aligned}
$$

where $\otimes$ means Kronecker product, $I_{2 n}=\operatorname{diag}\{1,1, \ldots, 1\}$, then, the distributed control protocol (6) and the disturbance observer (3) with $Y=P^{-1} Q$ can solve the flocking motion problem.

Proof. Distributed control protocol is continuous when $\delta(t-$ $\left.t_{k}\right) \equiv 1$. Let $\widetilde{q}_{i}(t)=q_{i}(t)-q_{r}(t)$, and $\widetilde{p}_{i}(t)=p_{i}(t)-p_{r}(t)$; it has $\dot{\tilde{q}}_{i}(t)=\widetilde{p}_{i}(t), \dot{\tilde{p}}_{i}(t)=\dot{p}_{i}(t)$. By calculating the control protocol (6), we can obtain

$$
\begin{aligned}
u_{i}(t)= & \sum_{j \in N_{i}} a_{i j} f\left(\left\|\widetilde{q}_{j}(t)-\widetilde{q}_{i}(t)\right\|\right) \vec{n}_{i j} \\
& +k \sum_{j \in N_{i}} a_{i j}\left(\widetilde{p}_{j}(t)-\widetilde{p}_{i}(t)\right)-\widehat{d}_{i}(t)-h_{i} c_{2} \widetilde{p}_{i}(t) .
\end{aligned}
$$

Define error variable $e_{w i}(t)=w_{i}(t)-\widehat{w}_{i}(t)=w_{i}(t)-z_{i}(t)+$ $Y p_{i}(t)$. The derivative of $e_{w i}(t)$ is written as

$$
\dot{e}_{w i}(t)=(A+Y C) e_{w i}(t) \text {. }
$$

Because of $\dot{\tilde{p}}_{i}(t)=u_{i}(t)+d_{i}(t)$, it has

$$
\begin{aligned}
\dot{\tilde{p}}_{i}(t)= & \sum_{j \in N_{i}} a_{i j} f\left(\left\|\widetilde{q}_{j}(t)-\tilde{q}_{i}(t)\right\|\right) \vec{n}_{i j} \\
& +k \sum_{j \in N_{i}} a_{i j}\left(\widetilde{p}_{j}(t)-\widetilde{p}_{i}(t)\right)+C e_{w i}(t) \\
& -h_{i} c_{2} \widetilde{p}_{i}(t) .
\end{aligned}
$$

Suppose the energy function is

$$
\begin{aligned}
V(t)= & \sum_{i=1}^{n} \sum_{j \in N_{i}} F\left(\left\|\widetilde{q}_{j}(t)-\widetilde{q}_{i}(t)\right\|\right)+\frac{1}{2} \sum_{i=1}^{n} \widetilde{p}_{i}^{T}(t) \widetilde{p}_{i}(t) \\
& +\sum_{i=1}^{n} e_{w i}{ }^{T}(t) P e_{w i}(t) .
\end{aligned}
$$

The derivative of $V(t)$ can be calculated:

$$
\begin{aligned}
\dot{V}(t)= & \sum_{i=1}^{n} \sum_{j \in N_{i}}-\widetilde{p}_{i}^{T}(t) f\left(\left\|\widetilde{q}_{j}(t)-\widetilde{q}_{i}(t)\right\|\right) \vec{n}_{i j} \\
& +\sum_{i=1}^{n} \widetilde{p}_{i}^{T}(t) \dot{\tilde{p}}_{i}(t)+\sum_{i=1}^{n} 2 e_{w i}(t)^{T} P \dot{e}_{w i}(t) .
\end{aligned}
$$

Putting $\dot{\tilde{p}}_{i}(t), \dot{e}_{w i}(t)$ into (12), it has

$$
\begin{aligned}
& \dot{V}(t)=\sum_{i=1}^{n}\left[\tilde{p}_{i}^{T}(t) k \sum_{j \in N_{i}} a_{i j}\left(\widetilde{p}_{j}(t)-\widetilde{p}_{i}(t)\right)\right. \\
& +\widetilde{p}_{i}^{T}(t) C e_{w i}(t)-h_{i} \mathcal{c}_{2} \widetilde{p}_{i}^{T}(t) \widetilde{p}_{i}(t) \\
& \left.+2 e_{w i}{ }^{T}(t) P(A+Y C) e_{w i}(t)\right] .
\end{aligned}
$$

Let $Q=P Y$ and $H=\operatorname{diag}\left(h_{1}, h_{2}, \ldots, h_{n}\right)$. Moreover, $n$ agents move in the two-dimensional Euclidean space; further consolidation can obtain

$$
\begin{aligned}
\dot{V}(t)=-\widetilde{p}^{T}(t)\left(I_{2} \otimes\left(k L+c_{2} H\right)\right) \widetilde{p}(t)+\frac{1}{2} \widetilde{p}^{T}(t) \\
\cdot\left(I_{2 n} \otimes C+I_{2 n} \otimes C^{T}\right) e_{w}(t)+e_{w}{ }^{T}(t) \\
\cdot\left(I_{2 n} \otimes(P A+Q C)^{T}+I_{2 n} \otimes(P A+Q C)\right) \\
\cdot e_{w}(t),
\end{aligned}
$$

where $\widetilde{p}(t)=\left[\widetilde{p}_{1}{ }^{T}(t), \widetilde{p}_{2}{ }^{T}(t), \ldots, \widetilde{p}_{n}{ }^{T}(t)\right]^{T}$ and $e_{w}(t)=$ $\left[e_{w 1}^{T}(t), e_{w 2}{ }^{T}(t), \ldots, e_{w n}{ }^{T}(t)\right]^{T}$.

Let $\eta(t)=\left[\begin{array}{ll}\tilde{p}^{T}(t) & e_{w}{ }^{T}(t)\end{array}\right]^{T} ;$ thus

$$
\dot{V}(t)=\eta^{T}(t) \Theta \eta(t),
$$

where

$\Theta$

$$
=\left[\begin{array}{cc}
-I_{2} \otimes\left(k L+c_{2} H\right) & \frac{1}{2}\left(I_{2 n} \otimes C\right) \\
\frac{1}{2}\left(I_{2 n} \otimes C^{T}\right) & I_{2 n} \otimes\left(P A+Q C+(P A)^{T}+(Q C)^{T}\right)
\end{array}\right] .
$$


According to the condition equation (7) in Theorem 4, we can obtain $\dot{V}(t)<0$. Thus, we have $\lim _{t \rightarrow \infty} \widetilde{q}(t)=0$.

That is,

$$
\begin{aligned}
& \lim _{t \rightarrow \infty}\left(q_{i}(t)-q_{r}(t)\right)=0, \\
& \lim _{t \rightarrow \infty}\left(p_{i}(t)-p_{r}(t)\right)=0 .
\end{aligned}
$$

Then, flocking motion will be eventually achieved in the interferential environment with control protocol (6).

Theorem 5. Supposen identical agents are affected by external disturbances. If the linear matrix inequality (7) and $0<c_{2}<2$ are satisfied, the system can achieve the flocking motion with the impulsive control protocol (6). Moreover, the movement is independent of the impulse period.

Proof. According to the integral feature of the Dirac function, distributed impulsive control protocol is described as

$$
\begin{aligned}
u_{i}(t)= & \sum_{j \in N_{i}} a_{i j} f\left(\left\|\widetilde{q}_{j}(t)-\widetilde{q}_{i}(t)\right\|\right) \vec{n}_{i j} \\
& +k \sum_{j \in N_{i}} a_{i j}\left(\widetilde{p}_{j}(t)-\widetilde{p}_{i}(t)\right)-\widehat{d}_{i}(t) \\
& -h_{i} \mathcal{c}_{2} \widetilde{p}_{i}(t) \delta\left(t-t_{k}\right) .
\end{aligned}
$$

And the dynamics of $i$ th agent has the following form with impulsive control protocol (6):

$$
\begin{aligned}
& \tilde{q}_{i}\left(t_{k}^{+}\right)-\tilde{q}_{i}\left(t_{k}^{-}\right)=0 \quad t=t_{k}, \\
& \tilde{p}_{i}\left(t_{k}^{+}\right)-\widetilde{p}_{i}\left(t_{k}^{-}\right)=-h_{i} \mathcal{c}_{2} \widetilde{p}_{i}\left(t_{k}^{-}\right) \quad t=t_{k}, \\
& \dot{\tilde{p}}_{i}(t) \\
& \quad=\sum_{j \in N_{i}} a_{i j} f\left(\left\|\widetilde{q}_{j}(t)-\tilde{q}_{i}(t)\right\|\right) \vec{n}_{i j} \\
& \quad+k \sum_{j \in N_{i}} a_{i j}\left(\tilde{p}_{j}(t)-\widetilde{p}_{i}(t)\right)+C e_{w i}(t) \quad t \neq t_{k} .
\end{aligned}
$$

At the moment of $t_{k}$, the energy function equation (11) can be rewritten as

$$
\begin{aligned}
V\left(t_{k}^{+}\right)= & \sum_{i=1}^{n} \sum_{j \in N_{i}} F\left(\left\|\widetilde{q}_{j}\left(t_{k}^{+}\right)-\widetilde{q}_{i}\left(t_{k}^{+}\right)\right\|\right) \\
& +\frac{1}{2} \sum_{i=1}^{n} \widetilde{p}_{i}^{T}\left(t_{k}^{+}\right) \widetilde{p}_{i}\left(t_{k}^{+}\right) \\
& +\sum_{i=1}^{n} e_{w i}^{T}\left(t_{k}^{+}\right) P e_{w i}\left(t_{k}^{+}\right) .
\end{aligned}
$$

Submitting (19) into (20), the difference of $V\left(t_{k}^{+}\right)$at the impulse moment $t_{k}$ can be calculated:

$$
\begin{aligned}
V\left(t_{k}^{+}\right)=\sum_{i=1}^{n} \sum_{j \in N_{i}} F\left(\left\|\widetilde{q}_{j}\left(t_{k}^{-}\right)-\widetilde{q}_{i}\left(t_{k}^{-}\right)\right\|\right)+\sum_{i=1}^{n} e_{w i}\left(t_{k}^{-}\right)^{T} P \\
\cdot e_{w i}\left(t_{k}^{-}\right)+\frac{1}{2} \sum_{i=1}^{n}\left(\widetilde{p}_{i}\left(t_{k}^{-}\right)-h_{i} c_{2} \widetilde{p}_{i}\left(t_{k}^{-}\right)\right)^{T} \\
\cdot\left(\widetilde{p}_{i}\left(t_{k}^{-}\right)-h_{i} c_{2} \widetilde{p}_{i}\left(t_{k}^{-}\right)\right) .
\end{aligned}
$$

Equation (22) is easy to obtain:

$$
V\left(t_{k}^{+}\right)-V\left(t_{k}^{-}\right)=\frac{1}{2} \sum_{i=1}^{n}\left[\widetilde{p}_{i}^{T}\left(t_{k}^{-}\right) h_{i} c_{2}\left(c_{2}-2\right) \tilde{p}_{i}\left(t_{k}^{-}\right)\right] .
$$

According to the condition $0<c_{2}<2$ in Theorem 5, $V\left(t_{k}^{+}\right)-V\left(t_{k}^{-}\right)<0$ is easy to be obtained. Thus, we have $\lim _{t \rightarrow \infty} \widetilde{q}(t)=0$; that is,

$$
\begin{aligned}
& \lim _{t \rightarrow \infty}\left(q_{i}(t)-q_{r}(t)\right)=0, \\
& \lim _{t \rightarrow \infty}\left(p_{i}(t)-p_{r}(t)\right)=0 .
\end{aligned}
$$

Therefore, flocking motion will be eventually achieved in the interferential environment with control protocol (6). Distributed impulsive control can solve the flocking motion of multiagent systems with exogenous disturbances, and the speeds of all agents will asymptotically converge to that of the leader.

\section{Simulation Examples}

Suppose that there are four identical agents with the topology in Figure 1 where only the first agent can receive information from the leader. Let control gain $k=1$ and balanced distance $d=2$ in the multiagent systems. The leader and four agents' initial positions are randomly generated within $\left[\begin{array}{llll}0 & 10 ; & 0 & 10\end{array}\right]$ and the initial velocities are generated in the range of $\left[\begin{array}{ll}0 & 1\end{array}\right]$ randomly. In addition, the initial velocity of the leader is $p_{r x}=0.5, p_{r y}=0.7$. The parameters of 

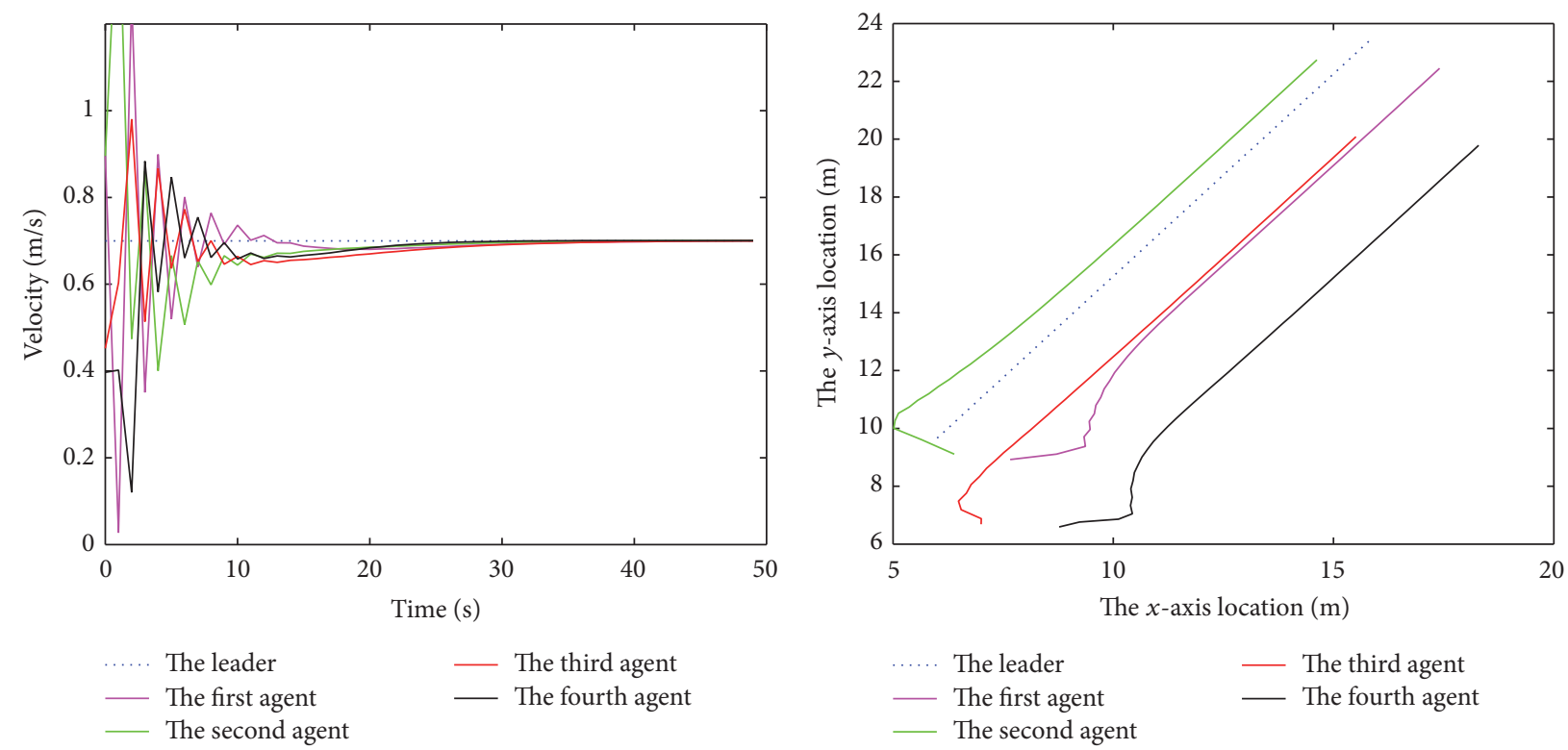

FIGURE 2: Velocities and relative positions of agents with disturbance observer.
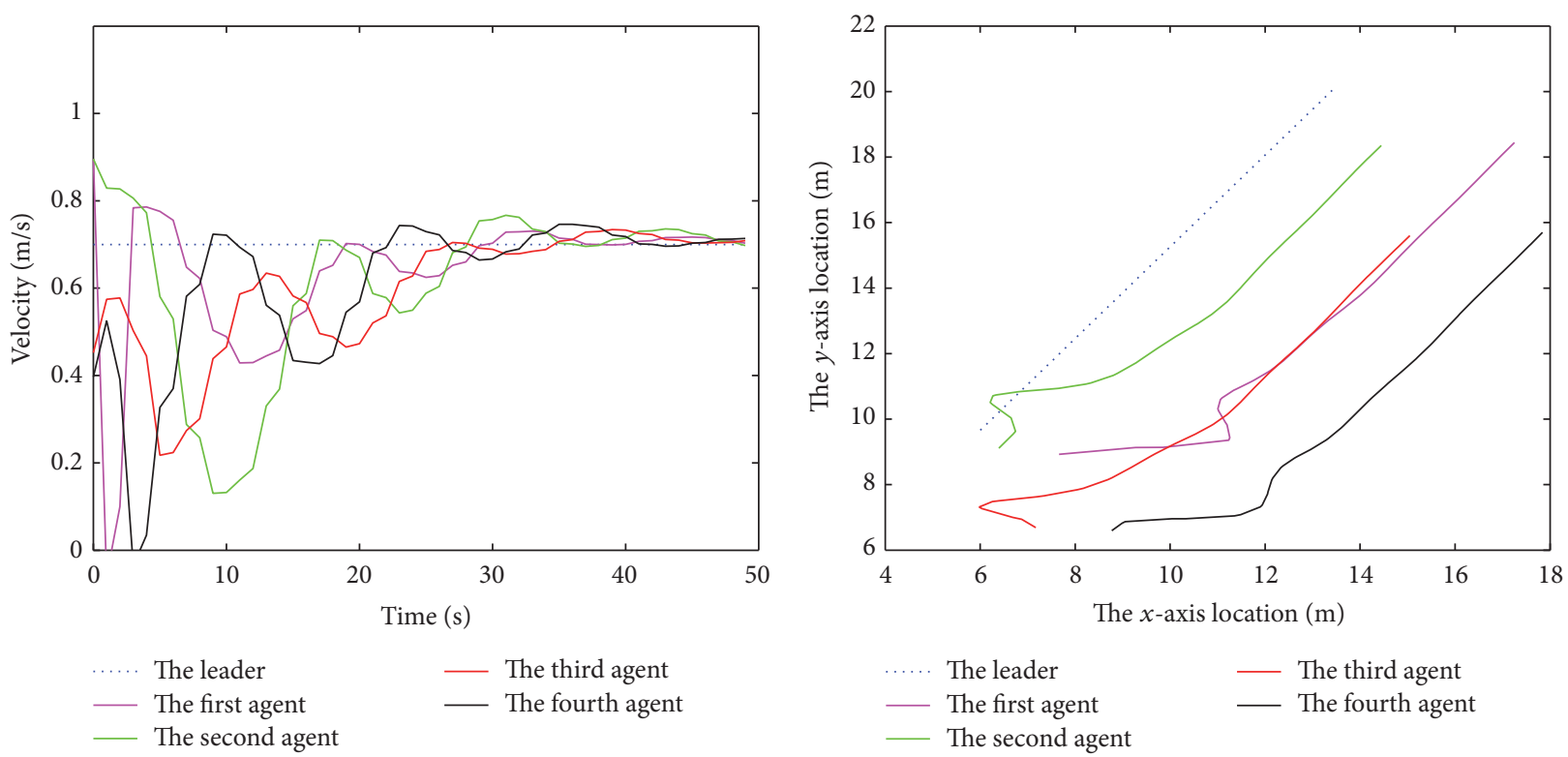

FIGURE 3: Velocities and relative positions of agents with impulsive control protocol.

exogenous disturbance subsystem are set as $A=\left[\begin{array}{cc}0 & 2 \\ -2 & 0\end{array}\right], C=$ $\left[\begin{array}{ll}1 & 0\end{array}\right]$. According to the sufficient condition of Theorem 4, we can get the unknown matrixes $P=\left[\begin{array}{cc}6 & -3 \\ -3 & 4\end{array}\right]$ and $Y=\left[\begin{array}{l}-2 \\ -1\end{array}\right]$.

4.1. Systems with Control Protocol $\delta\left(t-t_{k}\right) \equiv 1$. Let feedback coefficient $c_{2}=1$ in control protocol. Velocities and relative positions of agents are described in Figure 2 with the disturbance-observer-based-control protocol. Simulation results show that four agents can reach same speed and their relative positions have achieved consensus in $60 \mathrm{~s}$. The simulation results are the same as results without disturbances in
[5]; that is to say, the distributed control protocol is feasible for multiagent system with disturbances.

4.2. Systems with Impulsive Control Protocol. When the form of control protocol is impulse, and the system is affected by exogenous disturbances, the velocities and relative positions of four agents are illustrated in Figure 3 when $T=0.6 \mathrm{~s}$ and $c_{2}=1$. The flocking motion is completed with impulsive control method (6). Moreover, the convergence times become longer with more tortuous trajectories than that in above simulation with control protocol $\delta\left(t-t_{k}\right) \equiv 1$. 


\section{Conclusions}

In this paper, flocking motion problem of multiple-agent system with external dynamic disturbances is studied. The disturbance-observer-based distributed impulsive protocol is presented to solve the flocking motion of the multiagent systems. Simulation results reflect strong adaptability of the distributed impulsive control protocol in complex environment.

\section{Competing Interests}

The authors declare that they have no competing interests.

\section{Acknowledgments}

Project supported by the National Natural Science Foundation of China (Grants nos. 61273152, 61673200, and 61472172) and by the Natural Science Foundation of Shandong Province of China (Grant no. ZR2011FM017).

\section{References}

[1] E. Shaw, "Fish in schools," Natural History, vol. 84, pp. 40-45, 1975.

[2] C. W. Reynolds, "Flocks, herds, and schools: a distributed behavioral model," Computer Graphics, vol. 21, no. 4, pp. 25-34, 1987.

[3] T. Vicsek, A. Czirók, E. Ben-Jacob, I. Cohen, and O. Shochet, "Novel type of phase transition in a system of self-driven particles," Physical Review Letters, vol. 75, no. 6, pp. 1226-1229, 1995.

[4] W. Ren and R. W. Beard, "Consensus seeking in multiagent systems under dynamically changing interaction topologies," Institute of Electrical and Electronics Engineers. Transactions on Automatic Control, vol. 50, no. 5, pp. 655-661, 2005.

[5] R. Olfati-Saber, "Flocking for multi-agent dynamic systems: algorithms and theory," IEEE Transactions on Automatic Control, vol. 51, no. 3, pp. 401-420, 2006.

[6] H. G. Tanner, A. Jadbabaie, and G. J. Pappas, "Flocking in fixed and switching networks," IEEE Transactions on Automatic Control, vol. 52, no. 5, pp. 863-868, 2007.

[7] H. Su, X. Wang, and Z. Lin, "Flocking of multi-agents with a virtual leader," IEEE Transactions on Automatic Control, vol. 54, no. 2, pp. 293-307, 2009.

[8] H. Su, X. Wang, and W. Yang, "Flocking in multi-agent systems with multiple virtual leaders," Asian Journal of Control, vol. 10, no. 2, pp. 238-245, 2008.

[9] M. M. Zavlanos, A. Jadbabaie, and G. J. Pappas, "Flocking while preserving network connectivity," in Proceedings of the 46th IEEE Conference on Decision and Control (CDC '07), pp. 29192924, New Orleans, La, USA, December 2007.

[10] H. Su, X. Wang, and G. Chen, "Rendezvous of multiple mobile agents with preserved network connectivity," Systems \& Control Letters, vol. 59, no. 5, pp. 313-322, 2010.

[11] H. Su, X. Wang, and G. Chen, "A connectivity-preserving flocking algorithm for multi-agent systems based only on position measurements," International Journal of Control, vol. 82, no. 7, pp. 1334-1343, 2009.

[12] W.-H. Chen, "Disturbance observer based control for nonlinear systems," IEEE/ASME Transactions on Mechatronics, vol. 9, no. 4, pp. 706-710, 2004.
[13] L. Guo and W.-H. Chen, "Disturbance attenuation and rejection for systems with nonlinearity via DOBC approach," International Journal of Robust and Nonlinear Control, vol. 15, no. 3, pp. 109-125, 2005.

[14] X. Wei and L. Guo, "Composite disturbance-observer-based control and terminal sliding mode control for non-linear systems with disturbances," International Journal of Control, vol. 82, no. 6, pp. 1082-1098, 2009.

[15] X. Yao, L. Zhu, and L. Guo, "Disturbance-observer-based Control $\& H_{\infty}$ control for non-linear markovian jump singular systems with multiple disturbances," IET Control Theory \& Applications, vol. 8, no. 16, pp. 1689-1697, 2014.

[16] X. Yao and L. Guo, "Composite anti-disturbance control for Markovian jump nonlinear systems via disturbance observer," Automatica, vol. 49, no. 8, pp. 2538-2545, 2013.

[17] H. Yang, Z. Zhang, and S. Zhang, "Consensus of second-order multi-agent systems with exogenous disturbances," International Journal of Robust and Nonlinear Control, vol. 21, no. 9, pp. 945-956, 2011.

[18] H.-Y. Yang, L. Guo, and H.-L. Zou, "Robust consensus of multiagent systems with time-delays and exogenous disturbances," International Journal of Control, Automation and Systems, vol. 10, no. 4, pp. 797-805, 2012.

[19] J. Zhou, H. Zhang, L. Xiang, and Q. Wu, "Synchronization of coupled harmonic oscillators with local instantaneous interaction," Automatica, vol. 48, no. 8, pp. 1715-1721, 2012.

[20] Z.-H. Guan, Y. Wu, and G. Feng, "Consensus analysis based on impulsive systems in multiagent networks," IEEE Transactions on Circuits and Systems I: Regular Papers, vol. 59, no. 1, pp. 170178, 2012. 


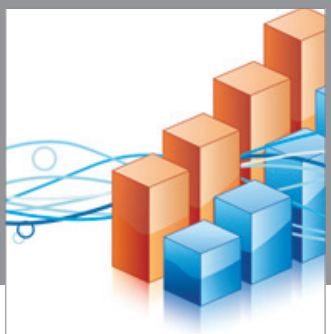

Advances in

Operations Research

vatem alat4

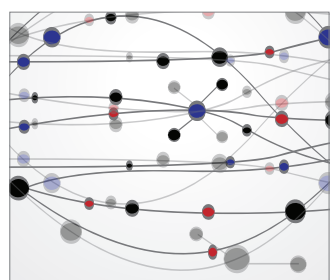

\section{The Scientific} World Journal
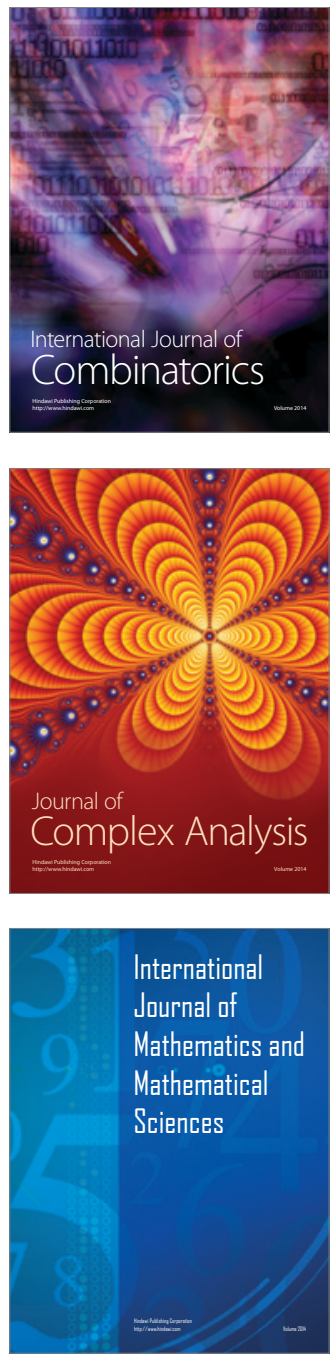
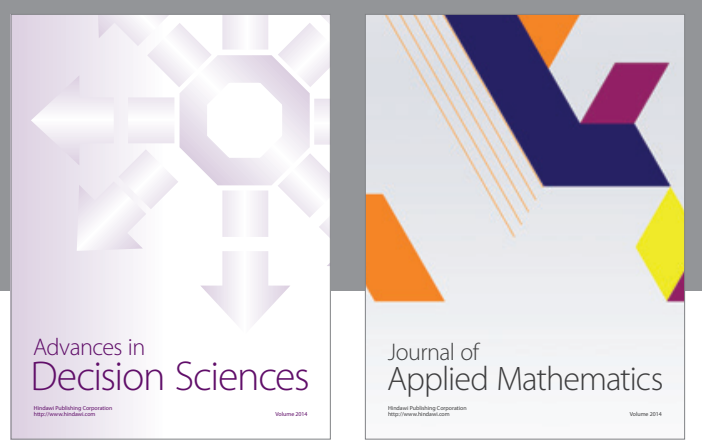

Algebra

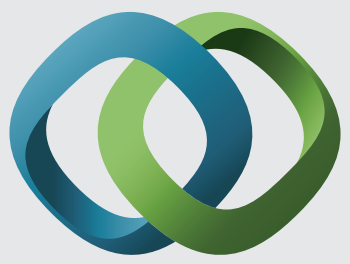

\section{Hindawi}

Submit your manuscripts at

https://www.hindawi.com
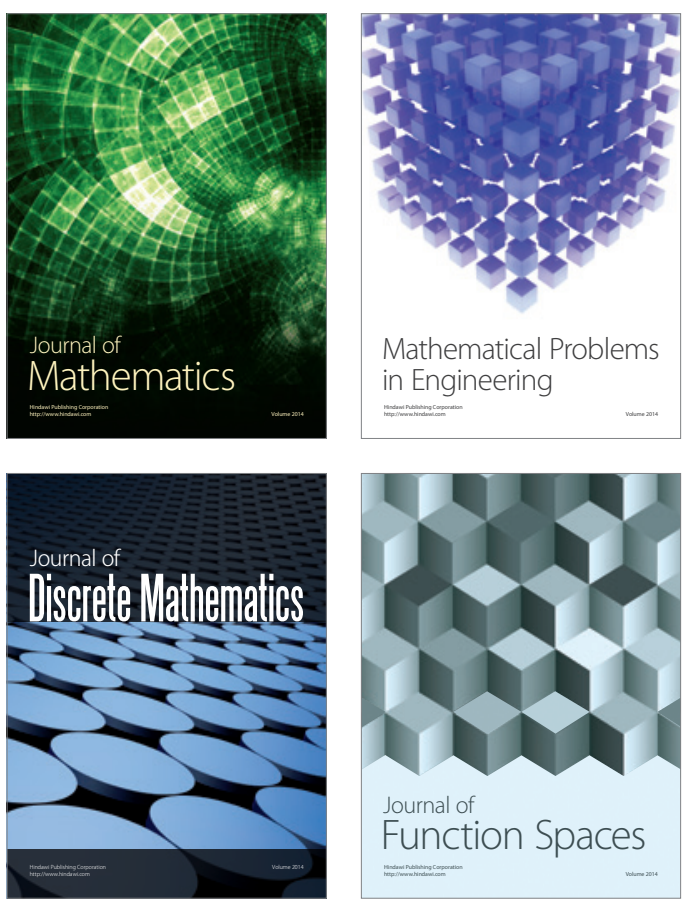

Mathematical Problems in Engineering
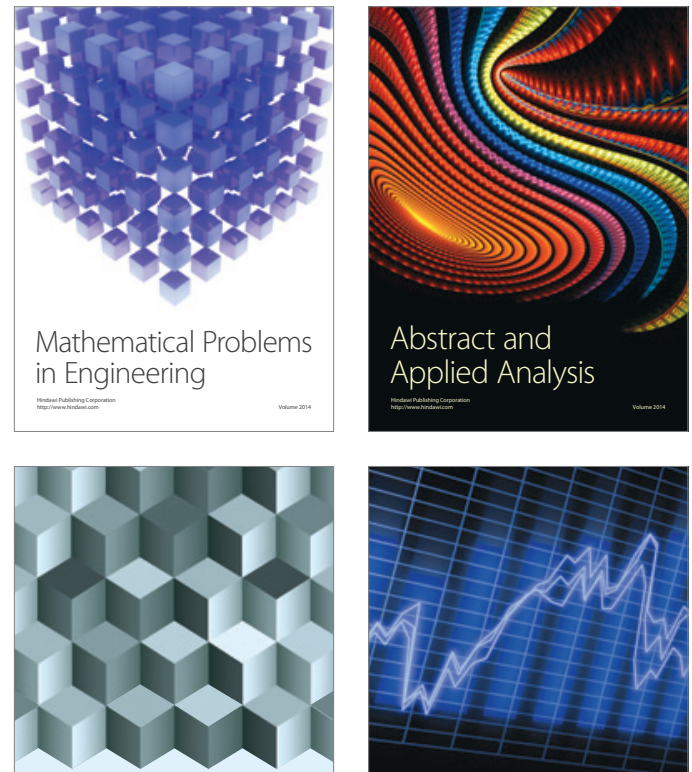

Journal of

Function Spaces

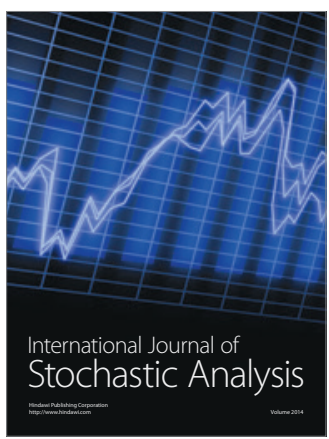

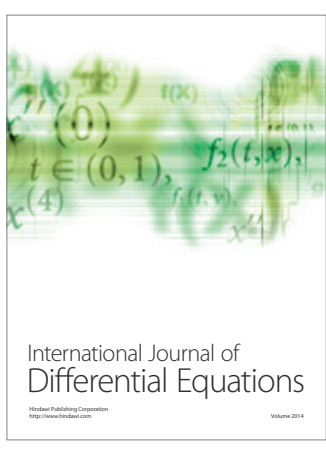
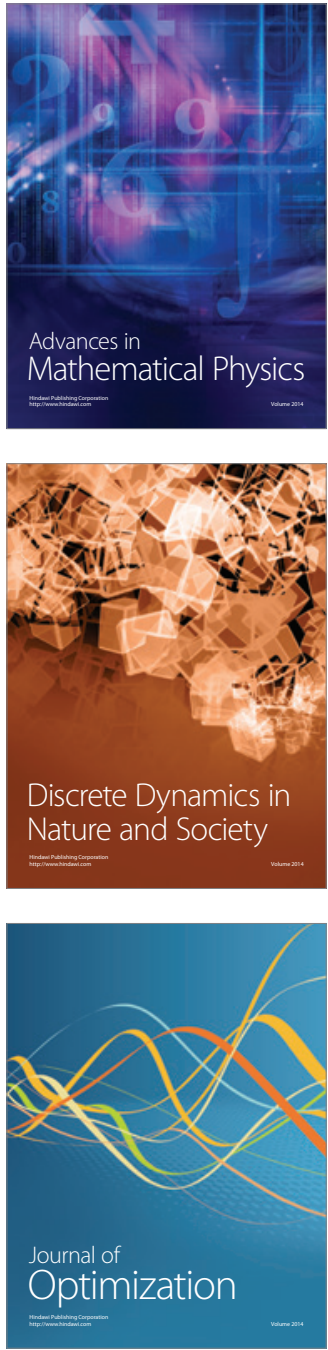